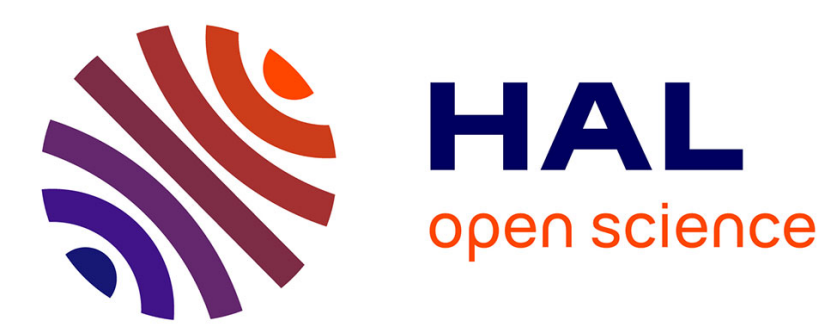

\title{
Ab Initio Molecular Dynamics of CdSe Quantum-Dot-Doped Glasses
}

Wenke Li, Xiujian Zhao, Chao Liu, François-Xavier Coudert

\section{To cite this version:}

Wenke Li, Xiujian Zhao, Chao Liu, François-Xavier Coudert. Ab Initio Molecular Dynamics of CdSe Quantum-Dot-Doped Glasses. Journal of the American Chemical Society, 2020, 142 (8), pp.3905-3912. 10.1021/jacs.9b12073 . hal-02492788

\section{HAL Id: hal-02492788 https://hal.science/hal-02492788}

Submitted on 27 Feb 2020

HAL is a multi-disciplinary open access archive for the deposit and dissemination of scientific research documents, whether they are published or not. The documents may come from teaching and research institutions in France or abroad, or from public or private research centers.
L'archive ouverte pluridisciplinaire HAL, est destinée au dépôt et à la diffusion de documents scientifiques de niveau recherche, publiés ou non, émanant des établissements d'enseignement et de recherche français ou étrangers, des laboratoires publics ou privés. 


\title{
Ab initio molecular dynamics of CdSe Quantum Dot-Doped Glasses \\ Wenke Li, ${ }^{1,2}$ Xiujian Zhao, ${ }^{1}$ Chao Liu,${ }^{1 *}$ François-Xavier Coudert ${ }^{2 *}$ \\ ${ }^{1}$ State Key Laboratory of Silicate Materials for Architectures, Wuhan University of \\ Technology, Hubei, 430070, China \\ ${ }^{2}$ Chimie ParisTech, PSL University, CNRS, Institut de Recherche de Chimie Paris, 75005 Paris, France
}

\begin{abstract}
We have probed the local atomic structure of the interface between the CdSe quantum dot (QD) and a sodium silicate glass matrix. Using ab initio molecular dynamics simulations, we determined the structural properties and bond lengths, in excellent agreement with previous experimental observations. Based on an analysis of radial distribution functions, coordination environment, and ring structures, we demonstrate that an important structural reconstruction occurs at the interface between the CdSe QD and the glass matrix. The incorporation of the CdSe QD disrupts the $\mathrm{Na}-$ $\mathrm{O}$ bonds, while stronger $\mathrm{SiO}_{4}$ tetrahedra are reformed. The existence of the glass matrix breaks the stable 4-member (4MR) and 6-member (6MR) of Cd-Se rings, and we observe a disassociated $\mathrm{Cd}$ atom migrated in the glass matrix. Besides, the formation of $\mathrm{Se}-\mathrm{Na}$ and $\mathrm{Cd}-\mathrm{O}$ linkages is observed at the $\mathrm{CdSe} \mathrm{QD} /$ glass interface. These results significantly extend our understanding of the interfacial structure of the CdSe QDdoped glasses, and provide physical and chemical insight into the possible defect structure origin of CdSe QD, of interest to the fabrication of the highly luminescent CdSe QD-doped glasses.
\end{abstract}

\section{Introduction}

The tunable optical and electronic properties of quantum dots induced by quantum confinement have stimulated enormous research interest. These properties were extensively explored for applications in the fields of lasers, ${ }^{1}$ light-emitting diodes $\left(\right.$ LEDs) ${ }^{2}$ and bio-labeling. ${ }^{3}$ Colloidal quantum dots exhibit excellent luminescence properties, however, agglomeration of these QDs in solution severely restricts their practical applications. As an alternative, quantum dot-doped glasses combine the good thermal and chemical stability of glass together with easy access to device fabrication, hence offering potential applications in non-linear optical devices ${ }^{4}$ and LEDs. ${ }^{5}$

Quantum dots were first fabricated in a glass matrix by Alexey Ekimov et al. in $1981 .^{6}$ L. E. Brus ${ }^{7}$ et al. first synthesized quantum dots in colloidal solutions in 1983. The past few decades have witnessed the blossoming of research in quantum dots, and chemically fabricated quantum dots can exhibit high photoluminescence quantum yield (PLQY) after surface passivation. Compared with their colloidal counterparts, quantum dots embedded in a glass matrix display poor PLQY, and the highest PLQY for CdSe 
embedded in glass have been measured at $3 \%{ }^{8}$ up to now. The enormous difference in the PLQY between QDs in colloidal solutions and in glass matrices naturally leads to the question: why is the quantum efficiency in the glass matrix so low? How can this situation be improved, to promote the practical applications of quantum dots? Experimentalists have worked for decades to shed light on these issues, and some mechanisms have been established to explain this phenomenon. It has been assumed that the presence of defects at the interface between QD and glass matrix can quench the excitonic emission and produce unfavorable defect emission, which is detrimental to applications. ${ }^{9}$ However, it is experimentally challenging to probe the origins of the interfacial defects and to characterize the structure and chemical environment of CdSe quantum dot-doped glass, due to the inadequate resolution of the existing techniques, the instability of the glass matrix under an electronic beam, and the relatively low concentration of QDs in the glass.

Theoretical modelling has been applied as an alternative to bring insight into these issues, and a comprehensive exploration of the local atomic structure can give guidance to the design of highly luminescent glasses. Computational materials studies have become very popular due to increasing computational power and the development of efficient numerical algorithms, and can investigate a system at the atomistic level which is not directly possible in experiments, gaining insight into both physical and chemical properties of materials. The two main computational methods for materials are classical approaches and $a b$ initio simulations. Both classical molecular dynamics (MD) and $a b$ initio molecular dynamics (AIMD) have been successfully used in modelling various multicomponent glass materials ${ }^{10-16}$ and CdSe nanocrystals. ${ }^{17-23}$ However, in spite of these extensive theoretical studies for these two separate systems, no atomistic calculations have been conducted so far on the composite QD/glass systems and their interfacial properties.

In this work, we have employed ab initio molecular dynamics in order to model CdSe QD embedded in a glass matrix, with a particular focus on the reconstruction occurring at the $\mathrm{QD} /$ glass interface. We started from classical MD calculations of the glass matrix, and quantum chemical models of the quantum dots. Because the interaction between the CdSe QD and glass matrix cannot be described accurately in classical MD due to the lack of the appropriate interatomic potentials, we used $a b$ initio methods. Our $a b$ initio MD methodology is based on quantum mechanics at density functional theory (DFT) level, which means it does not rely on a fixed functional form for interatomic interactions, but the electronic degrees of freedom of each atom are fully modelled. Due to the limitation of computational power cost, we chose a model of the composite system with the composition of $60 \mathrm{Na}_{2} \mathrm{O}-120 \mathrm{SiO}_{2}-33 \mathrm{CdSe}$ to conduct the AIMD simulations. The radial distribution functions for $\mathrm{Si}-\mathrm{O}, \mathrm{Na}-\mathrm{O}, \mathrm{Cd}-\mathrm{Se}, \mathrm{Cd}-\mathrm{O}, \mathrm{Se}-\mathrm{Na}$, Se$\mathrm{Se}$ and $\mathrm{Cd}-\mathrm{Cd}$ were calculated to compare with experimental counterparts. The coordination environment and the ring structures were analyzed. The results 
demonstrates that enormous structural reconstruction happens simultaneously in QD and glass matrix, with creation of $\mathrm{Cd}-\mathrm{O}$ bonds and $\mathrm{Se}-\mathrm{Na}$ bonds at the interface. The incorporation of the $\mathrm{CdSe} \mathrm{QD}$ disrupts $\mathrm{Na}-\mathrm{O}$ bonds, while stronger $\mathrm{SiO}_{4}$ tetrahedra are reformed. The glass matrix contributes to great structural reconstruction at the external surface of the quantum dot, making it hard to maintain the bulk structure even at its core. The results reported here can give a better fundamental understanding of this complex system, and giving insight for the fabrication of highly luminescent glasses containing quantum dots.

\section{Computational methods}

\subsection{Generation of glass matrix structure}

The starting configuration for the glass matrix was generated by placing atoms randomly in a cubic simulation box. The total number of atoms for the glass was 540 $(120 \mathrm{Na}, 120 \mathrm{Si}$, and $300 \mathrm{O})$, with the simulation cell sizes $(a=b=c=19.383 \AA, \alpha=$ $\beta=\gamma=90^{\circ}$ ) kept constant throughout the simulation, giving the density consistent with experimental values $\left(\rho=2.492 \mathrm{~g} / \mathrm{cm}^{3}\right) .{ }^{15}$ Hard constraints were imposed to avoid unphysically small interatomic distances. An initial classical molecular dynamics simulation was performed using a partial-charge rigid-ion pairwise potential developed by Pedone et al., ${ }^{16}$ with the DL_POLY classic package. ${ }^{24}$ The Coulomb interactions were calculated using the Ewald summation method ${ }^{25}$ with a precision of $10^{-5}$, and a real-space cutoff for short-range interactions set to $7.6 \AA$. The Verlet algorithm was applied for the integration of the equations of motion with a timestep of $1 \mathrm{fs}$. The glass structures were generated using a melt-quenching approach, in the NVT ensemble at the target density from experimental data, using a Nosé-Hoover thermostat ${ }^{26-28}$ with relaxation time of $0.1 \mathrm{ps}$. The initial structure was heated up gradually in steps of 1000 $\mathrm{K}$ with a $60 \mathrm{ps} \mathrm{MD}$ run at each temperature from $300 \mathrm{~K}$ to $6000 \mathrm{~K}$. After equilibration of the liquid at $6000 \mathrm{~K}$ during $400 \mathrm{ps}$, the system was cooled gradually in steps of 500 $\mathrm{K}$ with a $60 \mathrm{ps} \mathrm{MD}$ run at each temperature from $6000 \mathrm{~K}$ to $300 \mathrm{~K}$. A further $200 \mathrm{ps}$ NVT simulation was carried out at $300 \mathrm{~K}$, together with a 200 ps NVE simulation in order to equilibrate the structure.

\subsection{Generation of CdSe quantum dot-doped glass structure}

The experimental mechanism for the formation of CdSe QDs in glass is based on the phase decomposition of oversaturated solid solution ${ }^{9}$ and the time scale of the growth of these nanocrystals is far beyond the current computational method. So it is difficult to simulate the nucleation and growth stages, and here we directly introduced the CdSe QD into the glass matrix rather than trying to model directly the melt-quenching process. The $\mathrm{Cd}_{33} \mathrm{Se}_{33}$ QD (with a diameter of $13 \AA$ ) obtained in our previous work ${ }^{29}$ was incorporated into the glass matrix, removing glass atoms so interatomic distances between the QD and the glass matrix were longer than $2.5 \AA$. The final composition was $39 \mathrm{Na}_{2} \mathrm{O}-78 \mathrm{SiO}_{2}-33 \mathrm{CdSe}$ (Figure 1a). The interatomic interactions in CdSe QD used a Lenard-Jones pairwise potential validated in the literature ${ }^{22-23}$ and the 
Lorentz-Berthelot combining rules were used for interactions between CdSe QD and the glass matrix. The whole structure was equilibrated at $1000 \mathrm{~K}$, first using $200 \mathrm{ps}$ of NVT dynamics and then 200 ps of NVE dynamics, initially with atoms from the CdSe QD frozen. The structure was then cooled gradually in steps of $50 \mathrm{~K}$ with a $60 \mathrm{ps} \mathrm{MD}$ run at each temperature from $1000 \mathrm{~K}$ to $500 \mathrm{~K}$, while keeping CdSe QD frozen. Subsequent cooling from $500 \mathrm{~K}$ to $300 \mathrm{~K}$ took place in steps of $10 \mathrm{~K}$, with all atoms allowed to move. Finally, the structure were further equilibrated at $300 \mathrm{~K}$ with a $200 \mathrm{ps}$ NVT dynamics, and a final 200 ps NVE dynamics (Figure 1b). This simulation methodology was followed after comparison with several other simulations, in order to allow full relaxation of the system (especially the glass at high temperature) while keeping the integrity of the quantum dot. We have explored several quenching methods of the whole structures and the size of the glass matrix has been altered to test the representativity of our current model, which can be found in Supporting Information.

\subsection{Ab initio molecular dynamics of CdSe quantum dot in glass matrix}

After equilibration of systems using the classical MD simulations described above, we used the resulting configurations as starting point for $a b$ initio modelling at the Density Functional Theory (DFT) level, using Kohn-Sham formulation as implemented in the $\mathrm{CP} 2 \mathrm{~K}$ code. ${ }^{30}$ Simulations were run at the Generalized Gradient Approximation level, employing the PBE exchange-correlation functional. ${ }^{31}$ The plane wave cutoff was set to $600 \mathrm{Ry}$. For $\mathrm{Na}, \mathrm{Cd}$, and $\mathrm{Se}$, we used short-range molecularly optimized double- $\zeta$ single polarized basis sets (DZVP-MOLOPT-SR-GTH), while for O and Si we used a double- $\zeta$ single polarized basis set (DZVP-MOLOPT-GTH). ${ }^{32-33}$ After an initial geometry optimization with DFT, the resulting relaxed structure (Figure 1c) was used as initial structure for the AIMD simulations. The structure was quenched from $500 \mathrm{~K}$ to $300 \mathrm{~K}$ in steps of $50 \mathrm{~K}$, with a total $10 \mathrm{ps}$ AIMD run at a time step of $2 \mathrm{fs}$. The production run was conducted in the NVT ensemble at $300 \mathrm{~K}$ for $10 \mathrm{ps}$.

\section{Results and discussion}

Our goal in this work is to analyze the nature of the QD/glass matrix interface, and to quantify the reconstruction that takes place. As such, our initial structure (Figure 1a) is a "naïve" view of the hybrid system, and somewhat representative of the simplistic models that are sometimes used in the existing literature. By visual inspection, large structural reconstructions occurred after equilibration of the system, regardless of whether it is the final configuration of classical MD simulations of the glass matrix relaxation (Figure 1b), the DFT geometry optimization (Figure 1c), or the final structure of AIMD simulations (Figure 1d). In particular, we clearly observed the influence of the matrix in the introduction of strong disorder in the quantum dot. For the original QD structure, the $\mathrm{Cd}$ atoms were either coordinated with $4 \mathrm{Se}$ atoms (in the core of the QD) or $3 \mathrm{Se}$ atoms (at its external surface). A more complex coordination of the $\mathrm{Cd}$ and Se atoms in the glass-embedded QD can be observed, where some of the $\mathrm{Cd}-\mathrm{Se}$ linkages were broken, the coordination rings opened up, and coordination 
between the QD and glass was observed. The fact that the QD, in its glass matrix, cannot maintain its pristine structure, which was different from most of the models studied in the existing literature of QD in solution. ${ }^{17,19}$

(a)

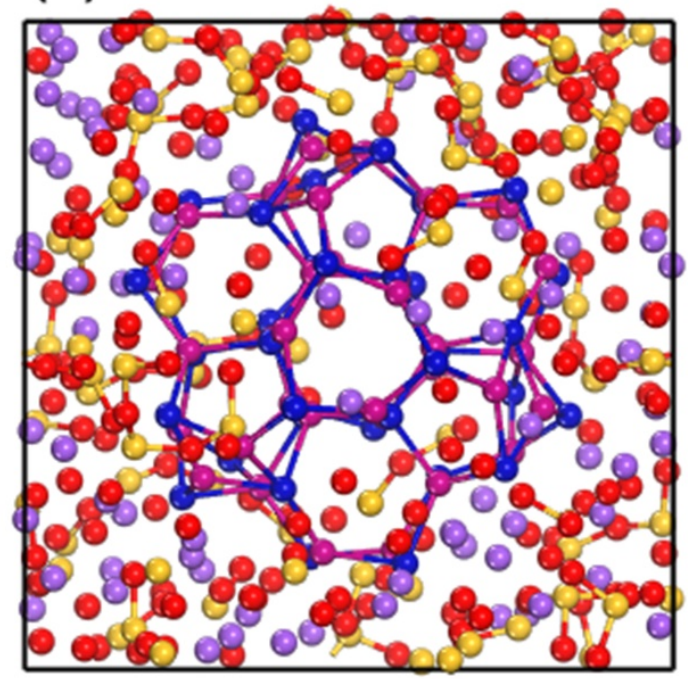

(c)

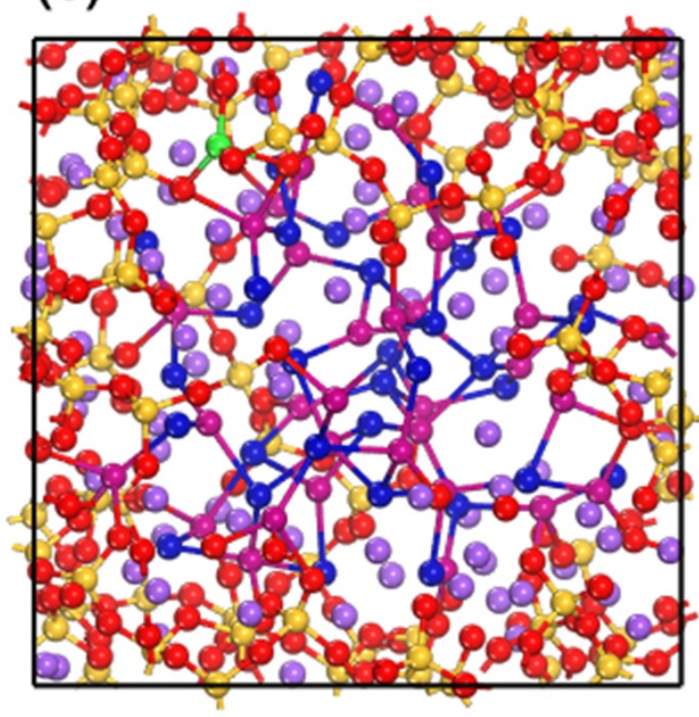

(b)

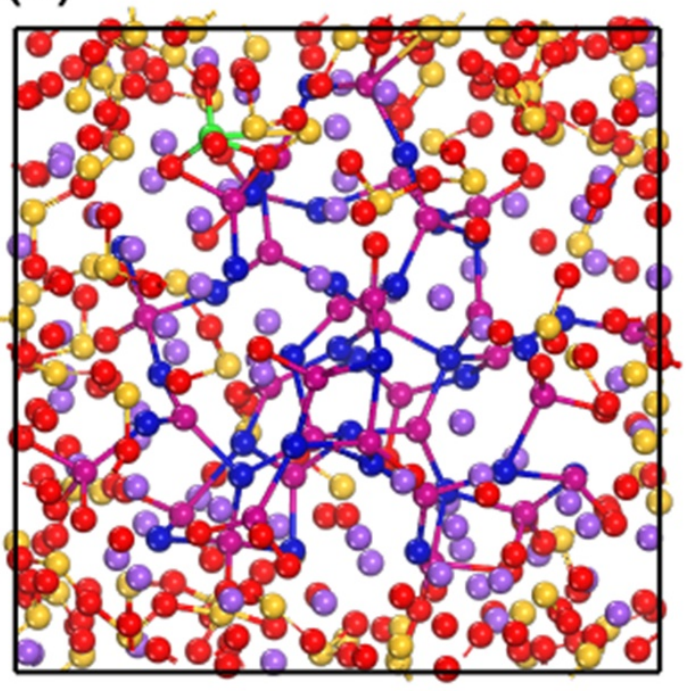

(d)

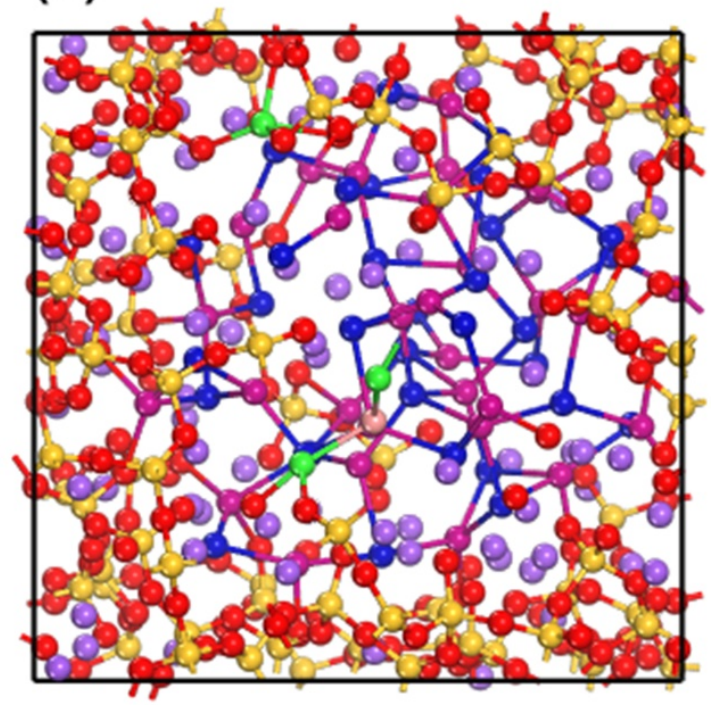

Figure 1. (a) The initial configuration for the classical MD calculations. (b) The final configuration for classical MD simulations, used as initial configuration for the geometry optimization by DFT method. (c) The geometry-optimized structure at the DFT level, used as the initial configuration for the ab initio molecular dynamics. (d) The final configuration from the AIMD simulations. Si: yellow, O: red, Na: purple, Se: magenta, $\mathrm{Cd}$ : blue. Se and $\mathrm{Cd}$ atoms dissociated from the quantum dot are highlighted in pink and green, respectively.

In addition to these changes in environment of the QD atoms, we observed some $\mathrm{Cd}$ ions from the QD surface that completely dissociated from the quantum dot structure, 
and migrated into the glass matrix (highlighted in green in Figure 1). This was observed for one atom during MD at the classical level, and confirmed in the AIMD with the migration of a second ion. Furthermore, during the ab initio MD we observed the dissociation of $\mathrm{Cd}-\mathrm{Se}-\mathrm{Cd}$ clusters (Se atom highlighted in pink). For the system size under study here, the percentages of dissociation were found to be $1.52 \%$ (Figure 1b and 1c) and 6.06\% (Figure 1d), respectively. These observations at the microscopic scale were novel, but they were in good agreement with experimental evidence of $\mathrm{Cd}$ atoms found to be dissolved in the matrix in as-prepared glass, seen by the extended Xray absorption fine structure spectroscopy analysis (EXAFS), ${ }^{34}$ in the CdSe QD-doped glass with the initial composition of $50 \mathrm{SiO}_{2}-20 \mathrm{~K}_{2} \mathrm{O}-20 \mathrm{ZnO}-5 \mathrm{~B}_{2} \mathrm{O}_{3}-1.5 \mathrm{CdO}-$ $1.5 \mathrm{CdS}-1.0 \mathrm{Na}_{2} \mathrm{SeO}_{3}(\mathrm{wt} \%)$, the $\mathrm{Cd}-\mathrm{O}$ contacts in all specimens with different thermal treatment were observed, attributed to the cadmium dissolved in the glass matrix.

We then analyzed the radial distribution functions for $\mathrm{Si}-\mathrm{O}$ (Figure 2a), $\mathrm{Na}-\mathrm{O}$ (Figure 2b), Cd-Se (Figure 2c) pairs. In each case, we compared RDFs for the initial structure (Figure 1a) and distribution functions obtained over the $a b$ initio molecular dynamics simulation. Compared with the initial structure, only minor changes in $\mathrm{Si}-\mathrm{O}$ and $\mathrm{Na}-\mathrm{O}$ interatomic distances were observed, with first-neighbor peaks at $1.64 \AA$ and $2.34 \AA$ respectively, consistent with the experimental value. ${ }^{13-14}$
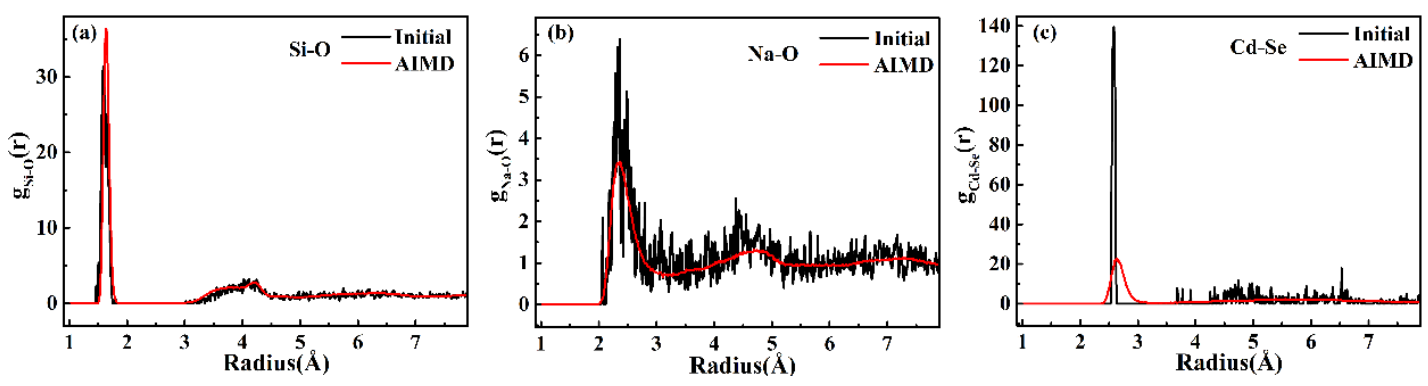

Figure 2. Radial distribution functions for (a) $\mathrm{Si}-\mathrm{O}$, (b) $\mathrm{Na}-\mathrm{O}$ and (c) $\mathrm{Cd}-\mathrm{Se}$ pairs in the initial structure (black) and averaged over the ab initio molecular dynamics (red), for a quantum dot embedded in the glass matrix.

Interestingly, an evolution in the Cd-Se distances was observed, from $2.59 \AA$ to a broader distribution with an average of $2.64 \AA$ ( $2 \%$ longer), due to both the finite temperature and the influence of the glass matrix, which weakens some of the $\mathrm{Cd}-\mathrm{Se}$ bonds. This influence of coordination was previously discussed in the literature. ${ }^{21,35-39}$ The Cd-Se bond length was $2.68 \AA$ when one Se atom was coordinated with $4 \mathrm{Cd}$ atoms, and $2.62 \AA$ when one Se atom was coordinated with $3 \mathrm{Cd}$ atoms in $\mathrm{Cd}_{33} \mathrm{Se}_{33}$ clusters. ${ }^{20}$ In this context, it was also interesting to note the size dependence of the $\mathrm{Cd}-\mathrm{Se}$ bond length, i.e., the smaller the QD's size, the shorter the Cd-Se bond length ${ }^{38-39}$ - while a lattice contraction was observed in the CdSe QD-doped glasses. ${ }^{40}$ This effect was linked to the surface/volume ratio, where smaller QDs had more surface atoms with 
shorted $\mathrm{Cd}-\mathrm{Se}$ bonds. We noted however in our $a b$ initio MD simulations, that the diameter of the QD in final structure was $16 \AA$, increased from $13 \AA$ in the initial structure. This demonstrates that the glass-embedded QD does not behave like the QD in vacuum: firstly, because of the chemical environment that the glass matrix constitutes, with glass/QD coordination. But other explanations have been advanced in the literature, it was suggested that the isotropic nature of the ordering of QDs in solution ${ }^{41}$ given rise to the strain effects between QDs and solution, resulting in the size-dependent and ligand dependent reconstruction of the QD surface. Moreover, the thermal and elastic mismatch between the nanocrystals and glass matrix can give rise to residual stresses upon cooling, ${ }^{42-44}$ increasing the extent of surface reconstruction.
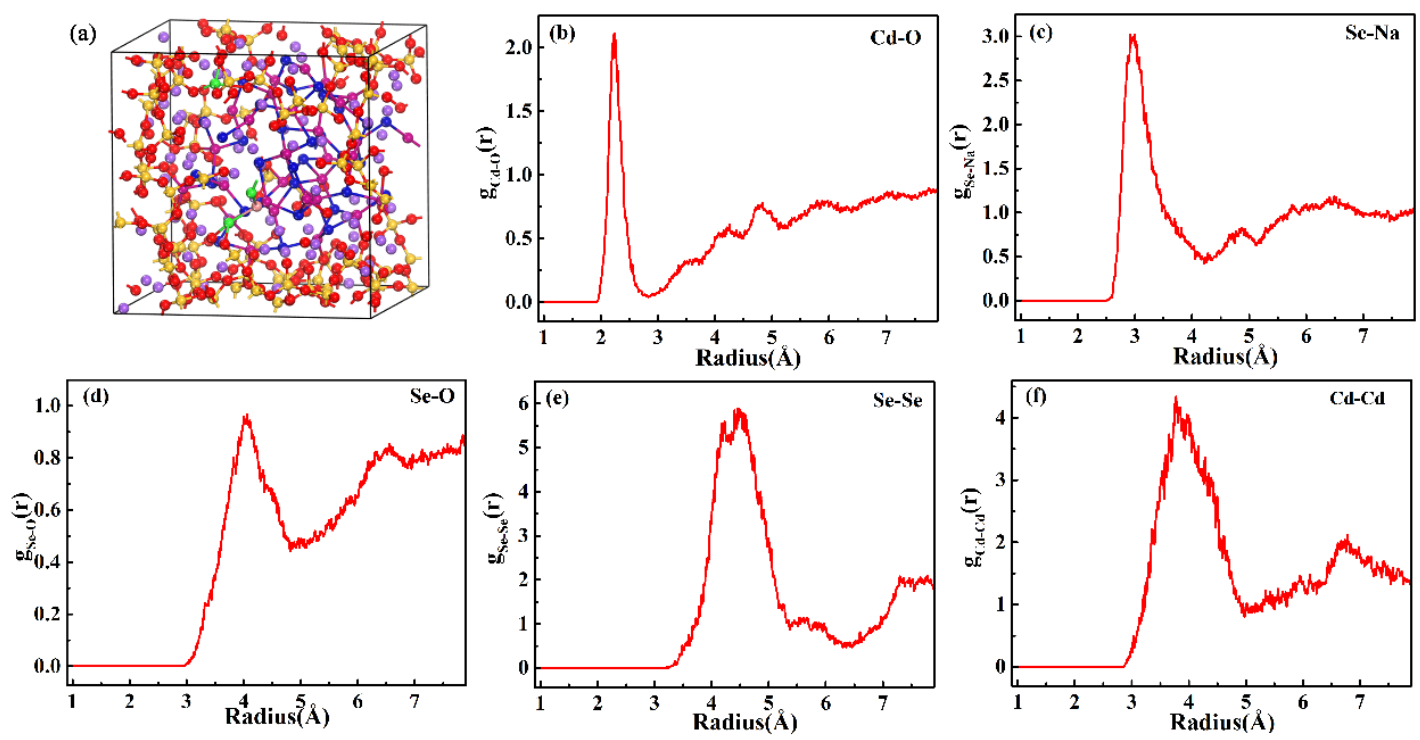

Figure 3. (a) The final configuration from the ab initio molecular dynamics. Radial distribution functions for (b) Cd-O, (c) Se-Na, (d) Se-O, (e) Se-Se, and (f) Cd-Cd pairs, averaged over the ab initio molecular dynamics (red), for a quantum dot embedded in the glass matrix.

We now turn our attention to the QD/glass interface (Figure 3a). Looking at the respective radial distribution functions, we observed the formation of $\mathrm{Cd}-\mathrm{O}$ and $\mathrm{Se}-\mathrm{Na}$ bonds. They were characterized by interatomic distance of $2.25 \AA$ (Figure 3b) and 2.91 $\AA$ (Figure 3c), respectively. These values, obtained from the ab initio molecular dynamics, were in excellent agreement with experimental data available. The interatomic $\mathrm{Cd}-\mathrm{O}$ distances were found to be $2.25 \AA$ based on the EXAFS characterization of CdSe QD-doped glasses, ${ }^{34}$ while the $\mathrm{Se}-\mathrm{Na}$ distances in the bulk $\mathrm{Na}_{2} \mathrm{Se}$ structure was $2.95 \AA$ (Crystallography Open Database ID: 9009065). From the experimental crystal lattice energies of $\mathrm{CdO}, \mathrm{CdSe}, \mathrm{Na}_{2} \mathrm{O}$ and $\mathrm{Na}_{2} \mathrm{Se}^{45}$ we expect the $\mathrm{Na}-\mathrm{Se}$ bond to be weaker than $\mathrm{Na}-\mathrm{O}$, and the $\mathrm{Cd}-\mathrm{O}$ bond to be stronger than $\mathrm{Cd}-\mathrm{Se}$. This can explain that the driving force for this reconstruction find its root in the $\mathrm{Cd}-\mathrm{O}$ interactions. On the other hand, $a b$ initio MD simulations confirm that there were no 
close contacts with formation Se-O (Figure 3d), Se-Se (Figure 3e) and Cd-Cd pairs (Figure 3f) at the QD/glass interface. The interatomic distances of these bonds were $3.92 \AA, 4.46 \AA$ and $3.70 \AA$, inconsistent with the experimental values, which were 1.83 $\AA,{ }^{29} 2.53 \AA^{34}$ and $4.20 \AA .{ }^{34}$

In order to understand the $\mathrm{QD} /$ glass interface in depth, the coordination environment (Table I) as well as the ring structures inside the $\mathrm{CdSe} \mathrm{QD} /$ glass systems were explored. We found that $52.40 \%$ of $\mathrm{O}$ atoms were bonded to $\mathrm{Na}$ atoms in the initial structure, while in the ab initio MD that number was $44.54 \%$. Meanwhile, there were opposite tendencies for the coordination between $\mathrm{O}$ and $\mathrm{Si}$ atom, with the percentages increased from $45.62 \%$ (initial structure) to $50.29 \%$ (ab initio MD). Moreover, while there was no $\mathrm{Cd}-\mathrm{O}$ bond in the initial structure, the AIMD showed $5.17 \%$ of $\mathrm{O}$ atoms were bonded with $\mathrm{Cd}$ atoms, confirming the formation of $\mathrm{Cd}-\mathrm{O}$ bonds in the interface.

Somewhat surprisingly, $100 \%$ of $\mathrm{Si}$ atoms were 4-coordinated with $\mathrm{O}$ atoms, while the coordination number of the $\mathrm{Si}$ atom with $\mathrm{O}$ atoms was 3.54 in the initial structure. This points to a dissymmetry in the $\mathrm{Si}-\mathrm{O}$ and $\mathrm{Na}-\mathrm{O}$ bonds of the glass, where the presence of the QD leads to disruption of the $\mathrm{Na}-\mathrm{O}$ bonds, while $\mathrm{Si}-\mathrm{O}$ coordination (and $\mathrm{Cd}-\mathrm{O}$, to a smaller extent) is kept intact, due to its stronger bond. In addition, the glass matrix also has a marked impact on the Cd-Se bonds in the QD. $23.72 \%$ of Se atoms were bonded with $\mathrm{Na}$ atoms and $27.70 \%$ of $\mathrm{Cd}$ atoms were bonded with $\mathrm{O}$ atoms in the $\mathrm{QD} /$ glass interface, with great decrease of the bonds between $\mathrm{Cd}$ atoms and Se atoms.

It is should be noted here that in our previous work, ${ }^{29}$ we studied a simple model of $\mathrm{Cd}_{33} \mathrm{Se}_{33}$ clusters capped with one sodium ion, and demonstrated that this results in the introduction of defect states in the HOMO-LUMO gap, giving rise to unusual nearinfrared luminescence. In addition, the HOMO and LUMO states were spread in the same region, indicating high probability of recombination of holes and electrons excited from these defect states. The results obtained in this work from ab initio MD show that when QD are surrounded by a glass matrix, the number of surrounding sodium ions within short distance (Na-Se linkages) is quite important. The defects at the interface are found to be not as simple as we expected in our previous work: a large percentage of Se atoms are bonded with $\mathrm{Na}$ atoms, in our glass where $\mathrm{Na}_{2} \mathrm{O}$ was introduced as glass modifier. The abundant nature of the sodium atoms in the glass matrix will greatly contribute to the defect emission, and a decrease in sodium content may diminish the number of interfacial defects, giving guidance to the design of glass composition in future work.

We further analyzed the details of the coordination numbers for $\mathrm{Si}-\mathrm{O}$ pairs (Figure 4a), $\mathrm{Na}-\mathrm{O}$ pairs (Figure 4b), Cd-Se pairs (Figure 4c) and $\mathrm{Se}-\mathrm{Cd}$ pairs (Figure 4d). These confirmed the strong rearrangement of the interface to promote the formation of $\mathrm{SiO}_{4}$ tetrahedra, which was also seen in the ring statistics (Table II) - with the occurrence 
of small ring sizes for $\mathrm{Si}-\mathrm{O}$ rings. For example, we observed 16 8-member rings (8MR) in the final structure, while no such ring was present in the initial configuration. Consistent with the data in Table I, the occurrence of higher coordination numbers of $\mathrm{Na}$ atoms with $\mathrm{O}$ atoms $(\mathrm{O} / \mathrm{Na}=6,5$, and 4) decreased while the frequency of lower coordination number for $\mathrm{Na}$ atoms with $\mathrm{O}$ atoms increased $(\mathrm{O} / \mathrm{Na}=3,2,1)$ compared with the initial structure (Figure 4b). Additionally, we observed a clear reduction of $\mathrm{Na}-\mathrm{O}$ rings, for all ring sizes but especially for the 4-member rings. This strengthened our conclusion that the rearrangement at the interface of the CdSe QD tends $\mathrm{Na}-\mathrm{O}$ bonds to maintain $\mathrm{Si}-\mathrm{O}$ coordination.

Table I. Average coordination number and percentages of $\mathrm{Na}, \mathrm{O}, \mathrm{Si}, \mathrm{Se}$ and $\mathrm{Cd}$ with different atoms in initial configuration, and averaged over the $a b$ initio MD simulation

\begin{tabular}{|c|c|c|c|c|c|}
\hline \multicolumn{2}{|c|}{ System } & \multicolumn{2}{|c|}{ Initial Structure } & \multicolumn{2}{|c|}{$A b$ initio MD } \\
\hline Atom & Bond & $\begin{array}{c}\text { Coordination } \\
\text { Number }\end{array}$ & Percentages & $\begin{array}{c}\text { Coordination } \\
\text { Number }\end{array}$ & Percentages \\
\hline \multirow{2}{*}{$\mathbf{N a}$} & $\mathrm{Na}-\mathrm{O}$ & 4.06 & $88.80 \%$ & 3.54 & $86.21 \%$ \\
\hline & Na-Se & 0.08 & $1.68 \%$ & 0.33 & $8.05 \%$ \\
\hline \multirow{3}{*}{$\mathbf{O}$} & O-Na & 1.63 & $52.40 \%$ & 1.42 & $44.54 \%$ \\
\hline & $\mathbf{O}-\mathbf{S i}$ & 1.42 & $45.62 \%$ & 1.60 & $50.29 \%$ \\
\hline & O-Cd & -- & - & 0.16 & $5.17 \%$ \\
\hline Si & Si-O & 3.54 & $87.64 \%$ & 4.00 & $100 \%$ \\
\hline \multirow{3}{*}{ Se } & $\mathrm{Se}-\mathrm{Cd}$ & 3.36 & $82.84 \%$ & 2.52 & $76.26 \%$ \\
\hline & $\mathbf{S e}-\mathbf{N a}$ & 0.18 & $4.48 \%$ & 0.78 & $23.72 \%$ \\
\hline & $\mathrm{Se}-\mathrm{O}$ & 0.36 & $8.96 \%$ & -- & -- \\
\hline \multirow{2}{*}{ Cd } & Cd-O & -- & - & 0.97 & $27.70 \%$ \\
\hline & $\mathrm{Cd}-\mathrm{Se}$ & 3.36 & $94.87 \%$ & 2.52 & $71.72 \%$ \\
\hline
\end{tabular}

The basic coordination environments of the CdSe QD are $\mathrm{CdSe}_{4}$ and $\mathrm{CdSe}_{3}$, whose counts were 12 and 21 in the initial structure, respectively. However, the average number of Se atoms around Cd dropped down, with counts for $\mathrm{CdSe}_{4}, \mathrm{CdSe}_{3}, \mathrm{CdSe}_{2}$, and $\mathrm{CdSe}_{1}$ of $6,11,12$, and 3 in the ab initio MD (Figure 4c), respectively. The total number of the $\mathrm{Cd}$ atoms bonded with $\mathrm{Se}$ atoms was 32 , with one $\mathrm{Cd}$ atom not bonded with any Se atom (migrated in the glass matrix, see Figure 1d). In terms of the coordination environment of Se atoms, all the Se atom were bonded with $\mathrm{Cd}$ atoms in the initial and final structure. The number of Se atoms with the $\mathrm{Cd} / \mathrm{Se}$ ratio $=4$ and 3 changed from 12 and 21, to 2 and 15 . In the same time, the appearance of the Se atoms with the ratio of $\mathrm{Cd} / \mathrm{Se}=2$ and $\mathrm{Cd} / \mathrm{Se}=1$ were observed (Figure 4d). The obvious changes in the ring size of $\mathrm{Cd}-\mathrm{Se}$ member rings confirmed the huge reconstruction of the quantum dot. The 4-member and 6-member $\mathrm{Cd}-\mathrm{Se}$ rings opened up, with only one 4-member and three 6-member $\mathrm{Cd}-\mathrm{Se}$ rings left in the final structure. The significant reconstruction of QD in this work distinguishes it from other work for the reason that 
the QDs almost keep the bulk structure in the center in other computer simulations. The common method to explore the QDs in solutions was to passivate the surface atom with limited number of organic ligands while the QD was incorporated into the inorganic glass matrix in our work. Only the surface atom were capped with organic ligands, without the simulation of the real chemical environment of the solution, leading to the smaller structural reconstruction compared to our work. Although there were some simulations that the QD was directly introduced into the solid matrix ${ }^{46}$ our work was aimed at the amorphous inorganic matrix for the first time, which constitutes a big part of research regarding the application of QDs for which the atomic structure and origin of defect emission have been rarely studied. The simulations in our work are closer to the real situation of a QD in a glass matrix, in which the matrix can have a more direct influence on the QD compared with the intended passivation of the QD. ${ }^{17},{ }^{19-20}$ Apart from the different initial structure building method, the difference in the bond nature between the inorganic atoms with QDs, such as glass modifiers $\mathrm{Na}$ atoms and nonbridging $\mathrm{O}$ atoms, and the organic ligand with QDs might have a relation to the different structure of QDs in glass matrix and solutions.
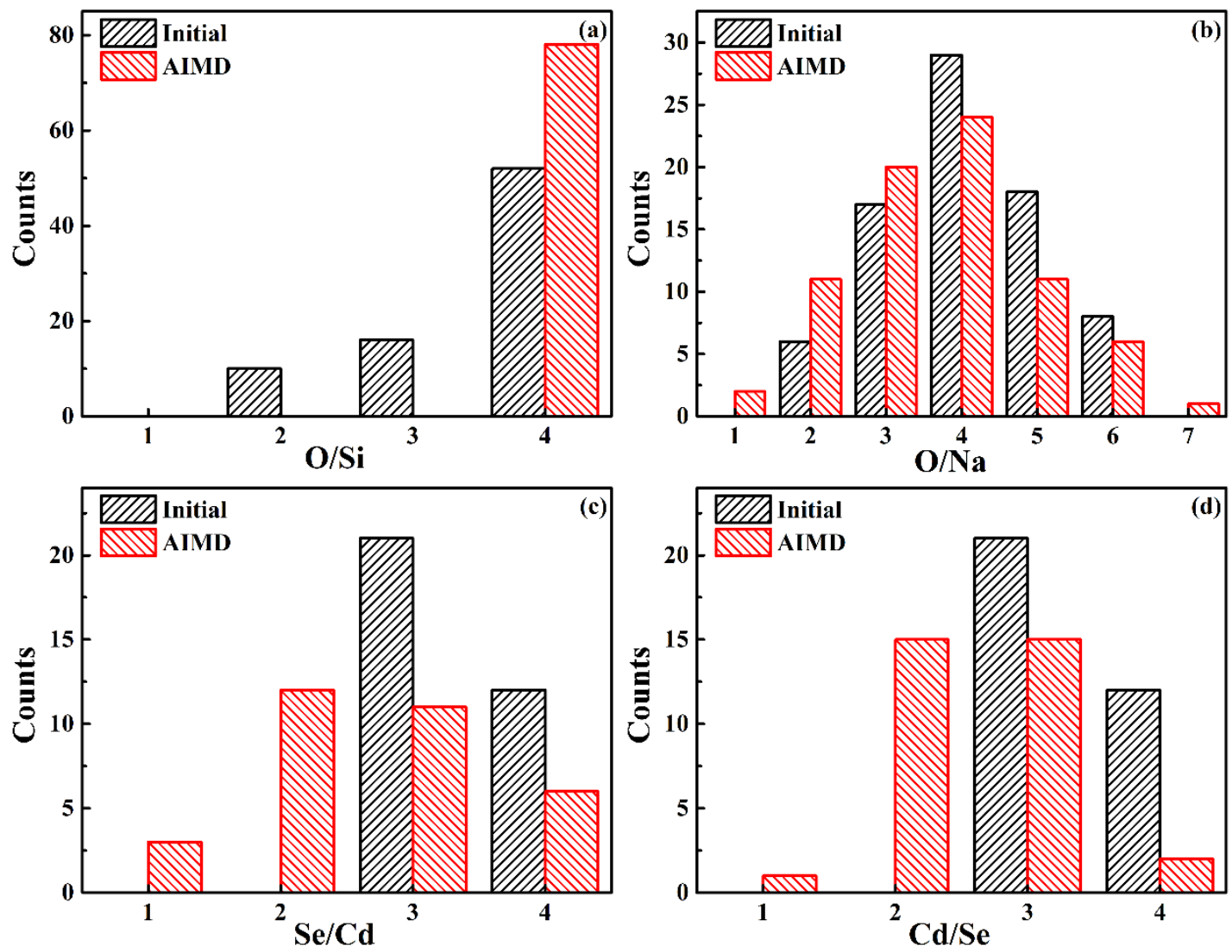

Figure 4. Histograms of coordination numbers for different pairs of atoms, comparing the initial structure (in black) and the average over the ab initio MD simulation (in red). (a) $\mathrm{O}$ coordination around $\mathrm{Si}$ atoms. (b) $\mathrm{O}$ coordination around $\mathrm{Na}$ atoms. (c) $\mathrm{Se}$ coordination around $\mathrm{Cd}$ atoms. (d) Cd coordination around $\mathrm{Se}$ atoms. 
Table II. The number of rings with different ring size for different ring members in initial structure and averaged over ab initio molecular dynamics.

\begin{tabular}{cccc}
\hline Ring Members & Ring Size & Initial Structure & Ab initio MD \\
\hline \multirow{4}{*}{ Si-O } & 8 & -- & 16 \\
& 10 & 3 & 10 \\
& 12 & -- & 4 \\
Na-O & 14 & 1 & 1 \\
& 16 & 1 & -- \\
\hline \multirow{5}{*}{ Cd-Se } & 4 & 88 & 45 \\
& 6 & 26 & 9 \\
& 8 & 16 & 2 \\
\hline & 4 & 12 & 1 \\
& 6 & 67 & 3 \\
& 10 & 6 & 7 \\
\hline
\end{tabular}

\section{Conclusions and perspectives}

To the best of our knowledge, our calculations are the first $a b$ initio molecular dynamics study of a CdSe quantum dot embedded in a glass matrix. Particularly, the structure of the CdSe QD-doped glass was obtained by the melt-quenching method, generated by the combination of the molecular dynamics and the $a b$ initio molecular dynamics. Distinct structural reconstructions were observed simultaneously in the glass matrix and CdSe QD. The incorporation of CdSe QD greatly disrupts the bonds between $\mathrm{Na}$ atoms and $\mathrm{O}$ atoms, with the reduction in the number of $\mathrm{O}$ atoms bonded with $\mathrm{Na}$ atoms, replaced by the $\mathrm{Si}$ atoms and $\mathrm{Cd}$ atoms. Unexpectedly, it also gives rise to the formation of the $\mathrm{SiO}_{4}$ tetrahedra and small ring size of $\mathrm{Si}-\mathrm{O}$ member rings, with all the $\mathrm{Si}$ atoms are 4-coordinated with the $\mathrm{O}$ atoms in the final structure. As for the interfacial structure, both the radial distribution function for the interatomic distance of $\mathrm{Cd}$ atoms and $\mathrm{O}$ atoms, Se atoms and $\mathrm{Na}$ atoms, as well as the analysis of the coordination environment of these atoms directly confirm the existence of the $\mathrm{Cd}-\mathrm{O}$ bonds and $\mathrm{Se}-\mathrm{Na}$ bonds, consistent with the experimental findings. Furthermore, it seems like that there are little possibilities of the formation of the Se-O bonds, Se-Se bonds, and $\mathrm{Cd}-\mathrm{Cd}$ bonds. In terms of the $\mathrm{CdSe} \mathrm{QD}$, the glass matrix have a greater influence on the reconstruction of the QD compared to QD in solutions. It is hard to maintain the stable 3-coordinated and 4-coordinated CdSe basic units when it is introduced into the glass matrix, with the 3-member and 4- member ring disappeared. Additionally, the disassociated $\mathrm{Cd}$ atoms and $\mathrm{Se}-\mathrm{Cd}-\mathrm{Se}$ clusters were observed to be migrated in the glass matrix. The complex structure of the glass matrix and the difference of the bond nature between the inorganic ions and QD might be attributed to the instability of QD in the glass matrix compared 
with the QD in organic solutions.

Our work gives an intuitive visual illustration of the local atomic interfacial structure of the CdSe QD-doped glass, promoting our understanding of the structural reconstructions of the glass matrix as well as the CdSe QD. Our results shed light on the impacts of the glass matrix on the $\mathrm{CdSe} \mathrm{QD}$, exploring the possible defect structure of the QD, thus can give guidance to the adjustment of the component of the glass matrix and to the fabrication of highly luminescent CdSe QD-doped glasses. Our work suggests it is worth adjusting the amount of sodium in the glass composition, which greatly contributes to the trap states in the band gap and leads to unfavorable defect emission. At the same time, we recommend reaching a compromise between the low sodium contents and melting temperature, because sodium can largely alter the melting point of glass. Besides, our results demonstrated that the surface $\mathrm{Cd}$ atoms are more likely to be passivated by the oxygen in the glass matrix, which may provide possibilities of fabricating core-shell CdSe QDs by adjusting the thermal treatment. The fabrication of a core-shell structure of CdSe QDs is more difficult and less explored compared with colloidal quantum dots. Work is in progress to extend our understanding of the electronic structure of these hybrid systems.

In our future work, various glass systems will be studied and we will explore the possible thermal strategies to synthesize core-shell CdSe quantum dots according to insight gained from $a b$ initio molecular dynamics. We also aim at studying more in depth the influence of the QD size on the nature of the QD/glass interface and the properties of the hybrid system. While the influence of QD size on its emission properties is well known for QDs in solution, the nature of the interface reconstruction for QDs embedded in glass matrices is not currently known. We expect, from the results obtained in this work, that the interfacial reconstruction remains similar: it is, after all, linked in large part not to the system size but to the nature of the competition between $\mathrm{Cd}-\mathrm{Se}, \mathrm{Cd}-\mathrm{O}$, and $\mathrm{Na}-\mathrm{O}$ interactions. However, because the surface/bulk ratio is directly linked to the size of the QD, the properties of the composite systems would be affected. While it is not currently possible to explore larger QD sizes at the level of theory chosen here ( $a b$ initio molecular dynamics), studies using QM/MM strategies could be of use in order to directly address the question of larger quantum dot sizes.

\section{Supporting Information}

Computational details of different quenching methods, computational details on different system sizes, additional discussion.

\section{Corresponding authors}

* hite@,whut.edu.cn; fx.coudert@,chimieparistech.psl.eu

\section{Acknowledgements}


This work was financially supported by the Natural Science Foundation of Hubei Province (2018CFA005), the Overseas Expertise Introduction Project (111 project) for Discipline Innovation of China (B18038), and the China Scholarship Council (201806950059). We acknowledge access to high-performance computing platforms provided by GENCI grant A0070807069.

\section{References}

1. Fan, F.; Voznyy, O.; Sabatini, R. P.; Bicanic, K. T.; Adachi, M. M.; McBride, J. R.; Reid, K. R.; Park, Y. S.; Li, X.; Jain, A.; Quintero-Bermudez, R.; Saravanapavanantham, M.; Liu, M.; Korkusinski, M.; Hawrylak, P.; Klimov, V. I.; Rosenthal, S. J.; Hoogland, S.; Sargent, E. H., Continuous-Wave Lasing in Colloidal Quantum Dot Solids Enabled by Facet-Selective Epitaxy. Nature 2017, 544 (7648), 75-79.

2. Yang, Y.; Zheng, Y.; Cao, W.; Titov, A.; Hyvonen, J.; Manders, J. R.; Xue, J.; Holloway, P. H.; Qian, L., High-Efficiency Light-Emitting Devices Based on Quantum Dots with Tailored Nanostructures. Nat. Photonics 2015, 9 (4), 259-266.

3. Wichner, S. M.; Mann, V. R.; Powers, A. S.; Segal, M. A.; Mir, M.; Bandaria, J. N.; DeWitt, M. A.; Darzacq, X.; Yildiz, A.; Cohen, B. E., Covalent Protein Labeling and Improved Single-Molecule Optical Properties of Aqueous CdSe/CdS Quantum Dots. ACS Nano 2017, 11 (7), 6773-6781.

4. Borrelli, N. F.; Smith, D. W., Quantum Confinement of PbS Microcrystals in Glass. J. Non-Cryst. Solids 1994, 180, 25-31.

5. Han, K.; Im, W. B.; Heo, J.; Chung, W. J., A Complete Inorganic Colour Converter Based on Quantum-Dot-Embedded Silicate Glasses for White Light-Emitting-Diodes. Chem. Commun. 2016, 52 (17), 3564-7.

6. Ekimov, A. I.; Onushchenko, A. A., Quantum Size Effect in Three-Dimensional Microscopic Semiconductor Crystals. JETP Lett. 1981, 34, 345-348.

7. Rossetti, R.; Nakahara, S.; Brus, L. E., Quantum Size Effects in The Redox Potentials, Resonance Raman Spectra, and Electronic Spectra of CdS Crystallites in Aqueous Solution. J. Chem. Phys. 1983, 79 (2), 1086-1088.

8. Han, K.; Yoon, S.; Chung, W. J., CdS and CdSe Quantum Dot-Embedded Silicate Glasses for LED Color Converter. Int. J. Appl. Glass Sci. 2015, 6 (2), 103-108.

9. Xia, M.; Liu, C.; Zhao, Z.; Wang, J.; Lin, C.; Xu, Y.; Heo, J.; Dai, S.; Han, J.; Zhao, X., Surface Passivation of CdSe Quantum Dots in All Inorganic Amorphous Solid by Forming Cd1-xZnxSe Shell. Sci. Rep. 2017, 7, 42359.

10. Ohkubo, T.; Tsuchida, E.; Deguchi, K.; Ohki, S.; Shimizu, T.; Otomo, T.; Iwadate, Y., Insights from $A b$ Initio Molecular Dynamics Simulations for a Multicomponent Oxide Glass. J. Am. Ceram. Soc. 2018, 101 (3), 1122-1134.

11. Fossati, P. C. M.; Rushton, M. J. D.; Lee, W. E., Atomic-Scale Description of Interfaces in Rutile/Sodium Silicate Glass-Crystal Composites. Phys. Chem. Chem. Phys. 2018, 20 (26), 17624-17636.

12. Deng, L.; Du, J., Effects of System Size and Cooling Rate on the Structure and Properties of Sodium Borosilicate Glasses from Molecular Dynamics Simulations. $J$. 
Chem. Phys. 2018, 148 (2), 024504.

13. Konstantinou, K.; Sushko, P. V.; Duffy, D. M., Modelling the Local Atomic Structure of Molybdenum in Nuclear Waste Glasses with Ab Initio Molecular Dynamics Simulations. Phys. Chem. Chem. Phys. 2016, 18 (37), 26125-26132.

14. Konstantinou, K.; Duffy, D. M.; Shluger, A. L., Structure and Luminescence of Intrinsic Localized States in Sodium Silicate Glasses. Phys. Rev. B 2016, 94 (17).

15. Ishii, Y.; Salanne, M.; Charpentier, T.; Shiraki, K.; Kasahara, K.; Ohtori, N., ADFTBased Aspherical Ion Model for Sodium Aluminosilicate Glasses and Melts. J. Phys. Chem. C 2016, 120 (42), 24370-24381.

16. Pedone, A.; Malavasi, G.; Menziani, M. C.; Cormack, A. N.; Segre, U., A New Self-Consistent Empirical Interatomic Potential Model for Oxides, Silicates and SilicaBased Glasses. J. Phys. Chem. B 2006, 110, 11780-11795.

17. Drijvers, E.; De Roo, J.; Martins, J. C.; Infante, I.; Hens, Z., Ligand Displacement Exposes Binding Site Heterogeneity on CdSe Nanocrystal Surfaces. Chem. Mater. 2018, 30 (3), 1178-1186.

18. Houtepen, A. J.; Hens, Z.; Owen, J. S.; Infante, I., On the Origin of Surface Traps in Colloidal II-VI Semiconductor Nanocrystals. Chem. Mater. 2017, 29 (2), 752-761.

19. Giansante, C.; Infante, I., Surface Traps in Colloidal Quantum Dots: A Combined Experimental and Theoretical Perspective. J. Phys. Chem. Lett. 2017, 8 (20), 5209-5215. 20. Del Ben, M.; Havenith, R. W. A.; Broer, R.; Stener, M., Density Functional Study on the Morphology and Photoabsorption of CdSe Nanoclusters. J. Phys. Chem. C 2011, 115 (34), 16782-16796.

21. Puzder, A.; Williamson, A. J.; Gygi, F.; Galli, G., Self-Healing of CdSe Nanocrystals: First-Principles Calculations. Phys. Rev. Lett. 2004, 92 (21), 217401.

22. Rabani, E., An Interatomic Pair Potential for Cadmium Selenide. J. Chem. Phys. 2002, $116(1)$.

23. Rabani, E., Structure and Electrostatic Properties of Passivated CdSe Nanocrystals. J. Chem. Phys. 2001, 115 (3), 1493-1497.

24. Smith, W.; Yong, C. W.; Rodger, P. M., DL_POLY: Application to Molecular Simulation. Mol. Simulat. 2010, 28 (5), 385-471.

25. Ewald, P. P., Die Berechnung Optischer und Elektrostatischer Gitterpotentiale. Annalen der physik 1921, (369), 253-287.

26. Bussi, G.; Donadio, D.; Parrinello, M., Canonical Sampling through Velocity Rescaling. J. Chem. Phys. 2007, 126 (1), 014101.

27. Hoover, W. G., Canonical Dynamics: Equilibrium Phase-Space Distributions. Phys. Rev. A Gen. Phys. 1985, 31 (3), 1695-1697.

28. Nosé, S., A Molecular Dynamics Method for Simulations in the Canonical Ensemble. Mol. Phys. 2006, 52 (2), 255-268.

29. Li, W.; Li, N.; Liu, C.; Greaves, G. N.; Ong, W. J.; Zhao, X., Understanding the Atomic and Electronic Structures Origin of Defect Luminescence of CdSe Quantum Dots in Glass Matrix. J. Am. Ceram. Soc. 2019, 102 (9), 5375-5385.

30. VandeVondele, J.; Krack, M.; Mohamed, F.; Parrinello, M.; Chassaing, T.; Hutter, 
J., Quickstep: Fast and Accurate Density Functional Calculations Using a Mixed Gaussian and Plane Waves Approach. Comput. Phys. Commun. 2005, 167 (2), 103-128. 31. Perdew, J. P.; Burke, K.; Ernzerhof, M., Generalized Gradient Approximation Made Simple. Phys. Rev. Lett. 1996, 78 (18), 3865-3868.

32. Goedecker, S.; Teter, M.; Hutter, J., Separable Dual-Space Gaussian Pseudopotentials. Phys. Rev. B 1996, 1703-1710.

33. VandeVondele, J.; Hutter, J., Gaussian Basis Sets for Accurate Calculations on Molecular Systems in Gas and Condensed Phases. J. Chem. Phys. 2007, 127, 114105. 34. Demourgues, A.; Greaves, G. N.; Bilsborrow, R.; Baker, G.; Sery, A.; Speit, B., XAFS Study of CdSe Quantum Dots in a Silicate Glass. Nucl. Instrum. Methods in Phys. Res. B 1995, 97, 166-168.

35. Sun, J.; Zheng, X.; He, H.; Chen, X.; Dong, B.; Fei, R., Theoretical Study of Ligand and Solvent Effects on Optical Properties and Stabilities of CdSe Nanoclusters. J. Mol. Struct. 2016, 1114, 123-131.

36. Wang, X.; Zeng, Q.; Shi, J.; Jiang, G.; Yang, M.; Liu, X.; Enright, G.; Yu, K., The Structure and Optical Absorption of Single Source Precursors for II-VI Quantum Dots. Chem. Phys. Lett. 2013, 568-569, 125-129.

37. Xu, S.; Wang, C.; Cui, Y., Theoretical Investigation of CdSe Clusters: Influence of Solvent and Ligand on Nanocrystals. J. Mol. Model. 2010, 16 (3), 469-73.

38. Chung, S. Y.; Lee, S.; Liu, C.; Neuhauser, D., Structures and Electronic Spectra of CdSe-Cys Complexes: Density Functional Theory Study of a Simple Peptide-Coated Nanocluster. J. Phys. Chem. B 2009, 113 (1), 292-301.

39. Jose, R.; Zhanpeisov, N. U.; Fukumura, H.; Baba, Y.; Ishikawa, M., StructureProperty Correlation of CdSe Clusters Using Experimental Results and First-Principles DFT Calculations. J. Am. Chem. Soc. 2006, 128, 629-626.

40. Hwang, Y.-N.; Shin, S.; Park, H. L.; Park, S.-H.; Kim, U., Effect of Lattice Contraction on the Raman Shifts of CdSe Quantum Dots in Glass Matrices. Phys. Rev. B 1996, 54 (21), 15120-15124.

41. Meulenberg, R. W.; Jennings, T.; Strouse, G. F., Compressive and Tensile Stress in Colloidal CdSe Semiconductor Quantum Dots. Phys. Rev. B 2004, 70, 235311.

42. Ballauff, M.; Brader, J. M.; Egelhaaf, S. U.; Fuchs, M.; Horbach, J.; Koumakis, N.; Kruger, M.; Laurati, M.; Mutch, K. J.; Petekidis, G.; Siebenburger, M.; Voigtmann, T.; Zausch, J., Residual Stresses in Glasses. Phys. Rev. Lett. 2013, 110 (21), 215701.

43. Kurkjian, C. R.; Gupta, P. K.; Brow, R. K., The Strength of Silicate Glasses: What Do We Know, What Do We Need to Know? Int. J. Appl. Glass Sci. 2010, 1 (1), 27-37. 44. Mastelaro, V. R.; Zanotto, E. D., Residual Stresses in a Soda-Lime-Silica GlassCeramic. J. Non-Cryst. Solids 1996, 194, 297-304.

45. Mu, L.; Feng, C., Topological Research on Lattice Energies for Inorganic Compounds. MATCH Commun. Math. Comput. Chem. 2006, 56, 97-111.

46. Giberti, F.; Voros, M.; Galli, G., Design of Heterogeneous Chalcogenide Nanostructures with Pressure-Tunable Gaps and without Electronic Trap States. Nano Lett. 2017, 17 (4), 2547-2553. 
TOC Graphic

$39 \mathrm{Na}_{2} \mathrm{O}-78 \mathrm{SiO}_{2}-33 \mathrm{CdSe}$

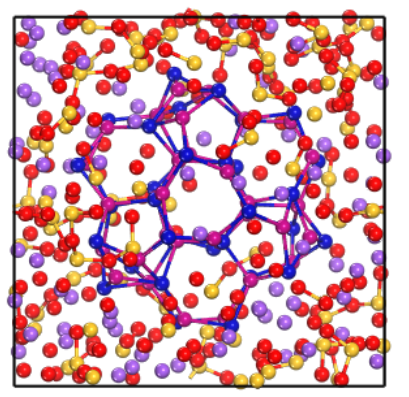

